Journal of Comparative Psychology

Social Environment Elicits Lateralized Behaviors in Gorillas (Gorilla gorilla gorilla) and Chimpanzees (Pan troglodytes)

Caterina Quaresmini, Gillian S. Forester, Caterina Spiezio, and Giorgio Vallortigara

Online First Publication, April 21, 2014. http://dx.doi.org/10.1037/a0036355

CITATION

Quaresmini, C., Forrester, G. S., Spiezio, C., \& Vallortigara, G. (2014, April 21). Social Environment Elicits Lateralized Behaviors in Gorillas (Gorilla gorilla gorilla) and Chimpanzees (Pan troglodytes). Journal of Comparative Psychology. Advance online publication. http://dx.doi.org/10.1037/a0036355 


\title{
Social Environment Elicits Lateralized Behaviors in Gorillas (Gorilla gorilla gorilla) and Chimpanzees (Pan troglodytes)
}

\author{
Caterina Quaresmini \\ University of Trento
}

Caterina Spiezio

Parco Natura Viva, Bussolengo, Italy

\author{
Gillian S. Forrester \\ University of Westminster \\ Giorgio Vallortigara \\ University of Trento
}

\begin{abstract}
The influence of the social environment on lateralized behaviors has now been investigated across a wide variety of animal species. New evidence suggests that the social environment can modulate behavior. Currently, there is a paucity of data relating to how primates navigate their environmental space, and investigations that consider the naturalistic context of the individual are few and fragmented. Moreover, there are competing theories about whether only the right or rather both cerebral hemispheres are involved in the processing of social stimuli, especially in emotion processing. Here we provide the first report of lateralized social behaviors elicited by great apes. We employed a continuous focal animal sampling method to record the spontaneous interactions of a captive zoo-living colony of chimpanzees (Pan troglodytes) and a biological family group of peer-reared western lowland gorillas (Gorilla gorilla gorilla). We specifically focused on which side of the body (i.e., front, rear, left, right) the focal individual preferred to keep conspecifics. Utilizing a newly developed quantitative corpus-coding scheme, analysis revealed both chimpanzees and gorillas demonstrated a significant group-level preference for focal individuals to keep conspecifics positioned to the front of them compared with behind them. More interestingly, both groups also manifested a population-level bias to keep conspecifics on their left side compared with their right side. Our findings suggest a social processing dominance of the right hemisphere for context-specific social environments. Results are discussed in light of the evolutionary adaptive value of social stimulus as a triggering factor for the manifestation of group-level lateralized behaviors.
\end{abstract}

Keywords: behavior, cerebral lateralization, chimpanzee, gorilla, social stimuli

Recent evidence of lateralized motor actions underpinned by contralateral neural regions in different vertebrate (MacNeilage, Rogers, \& Vallortigara, 2009; Rogers \& Andrew, 2002; Rogers,

Caterina Quaresmini, Center for Mind/Brain Sciences, University of Trento, Rovereto, Italy; Gillian S. Forrester, Department of Psychology, University of Westminster, London, England; Caterina Spiezio, Research and Conservation Department, Parco Natura Viva, Bussolengo, Italy; Giorgio Vallortigara, Center for Mind/Brain Sciences, University of Trento, Rovereto, Italy.

We thank Professor D.A. Leavens for assistance with data analysis and Professor K. Bard for suggestions on the interpretation of results. We also thank the Garda Zoological Park - Parco Natura Viva in Verona (Italy), and the Port Lympne Park in Kent (United Kingdom), for access to and video data of their groups of chimpanzees and gorillas, respectively. We gratefully acknowledge funding support provided by Provincia autonoma di Trento and Fondazione Cassa di Risparmio di Trento e Rovereto. GV was supported by an ERC Advanced Research Grant PREMESOR (Grant Agreement No. 295517).

Correspondence concerning this article should be addressed to Caterina Quaresmini, Department of Psychology and Cognitive Sciences, University of Trento, Corso Bettini 31, 38068 Rovereto, Italy. E-mail: caterina.quaresmini@gmail.com
Vallortigara, \& Andrew, 2013) and invertebrate species (Anfora et al., 2011; Frasnelli, Vallortigara, \& Rogers, 2012) suggests early evolution of cerebral lateralization across the animal kingdom. Cerebral lateralization has been associated with some advantages related to an increase in neural capacity that helps to decrease the duplication of function, enable, parallel processing and prevent the simultaneous initiation of incompatible responses (Levy, 1977; Rogers, 2000a; Rogers, Zucca, \& Vallortigara, 2004). The literature suggests an early division of labor for the two hemispheres, where the left hemisphere is dominant for the processing of relevant stimuli and learnt routine tasks (top-down control), whereas the right hemisphere is more involved in reacting to unexpected events and encoding the spatial relationships occurring in the surrounding environment (bottom-up control; MacNeilage et al., 2009). Therefore, a selective lateral positioning of the body toward the environmental stimuli can enhance the survival of the individual by facilitating motor activities (e.g., feeding, approach and avoidance behaviors, etc.) that are controlled by the dominant hemisphere for a specific task. Moreover, a strategic positioning might allow two cognitive tasks (e.g., food processing and conspecific vigilance) to take place at the same time (Rogers et al., 2004).

However, these advantages are not sufficient to explain why in many vertebrates and invertebrates asymmetrical behaviors are 
apparent at the population-level. Recent discoveries revealed that directional asymmetries are particularly evident in gregarious, rather than in solitary species. This suggests that the social environment might have played a key role in aligning individual-level asymmetries, to coordinate the behavior among individuals for predator defense and for cooperation (Ghirlanda, Frasnelli, \& Vallortigara, 2009; Ghirlanda \& Vallortigara, 2004; Vallortigara \& Rogers, 2005). Indeed, lateralized behaviors are particularly evident in response to social stimuli, such as individual recognition, decoding of other's intentions, and learning the social hierarchical system, which are asymmetrically processed at the neural level in many gregarious vertebrate species (for a review, see Rosa Salva, Regolin, Mascalzoni, \& Vallortigara, 2012).

In vertebrata a tendency exists to keep conspecifics in the left visual field (fishes: Sovrano, Bisazza, \& Vallortigara, 2001; toads: Robins, Lippolis, Bisazza, Vallortigara, \& Rogers, 1998; lizards: Deckel, 1995; Hews \& Worthington, 2001; pigeons: Nagy, Àkos, Biro, \& Vicsek, 2010; chicks: Vallortigara, 1992; Vallortigara \& Andrew, 1991; beluga whales: Karenina et al., 2010), suggesting a right hemisphere dominance for social responses. These lateralized perceptual behaviors might derive from an early delineation of hemisphere dominance for responding to predators' and preys' reaction (MacNeilage et al., 2009).

The right hemisphere of human and nonhuman animals is particularly dominant for face perception and social recognition. For example, a left gaze bias (LGB) for face perception (i.e., a looking time preference for exploring the left side of a centrally presented face) is apparent in humans (behavioral study: Burt \& Perret, 1997; neuro-imaging: Kanwisher, Tong, \& Nakayama, 1998; braindamaged patients: De Renzi, Perani, Carlesimo, Silveri, \& Fazio, 1994), chimpanzees (Morris \& Hopkins, 1993), rhesus monkeys (Guo, Meints, Hall, Hall, \& Mills, 2009), dogs (Guo et al., 2009), and sheep (Peirce, Leigh, \& Kendrick, 2000).

Recent studies report that both hemispheres may process social stimuli, but the level of complexity of the task as well as the motivational and emotional factors may drive a dominant role for one hemisphere over the other (see Rosa Salva et al., 2012). This division of labor is also evident at a higher cognitive level, that is, the processing and manifestation of emotions. The literature is divided between two prominent emotional processing theories: the right hemisphere hypothesis (Borod et al., 1998; Campbell, 1982), which asserts that the right hemisphere is uniquely involved in the processing of all type of emotions, and the valence hypothesis (Davidson, 1995), which claims that both the right and the left hemispheres are involved, but differently in relation to negative and positive emotions, respectively. Nevertheless, to date, no human studies concentrate on the actual physical positioning of individuals within their social network.

Nonhuman primates demonstrate a large repertoire of social activities (e.g., vocalizations, gestures, facial expressions, social learning). Although they represent an excellent animal model for the investigation of lateralized behaviors related to the social environment, the wider literature only expresses data regarding the production of asymmetrical facial expressions (marmosets: HookCostigan \& Rogers, 1998; macaques: Hauser, 1993; baboons: Wallez \& Vauclair, 2011; chimpanzees: Fernández-Carriba, Loeches, Morcillo, \& Hopkins, 2002), the display of lateralized self-directed behaviors during stressful conditions in chimpanzees (Leavens, Aureli, \& Hopkins, 2004) and during spontaneous ac- tivities in orang-utans (Rogers \& Kaplan, 1996). An overall righthemisphere dominance for the processing of social stimuli and the expression of emotive response was reported. Additionally, the assessment of eyedness in relation to different types of stimuli has been shown to reflect a right hemisphere advantage for emotive responses. For example, red-capped mangabeys showed a grouplevel bias for using the left more than the right eye when observing arousing stimuli in a monocular fashion (i.e., palatable food). However, only individual-level preferences emerged with neutral stimuli (i.e., unpalatable food; see de Latude, Demange, Bec, \& Blois-Heulin, 2009). More recently, Braccini, Lambeth, Schapiro, and Fitch (2012) assessed eye preference in captive chimpanzees, using pictures with a positive or negative valence. They found a hemispheric specialization for emotion processing with a left eye/ right hemisphere advantage for negative stimuli and right eye/left hemisphere advantage for positive stimuli. Although these studies do not seem to contradict either emotional processing hypotheses (i.e., right hemisphere and valence), both indicate that the right hemisphere is heavily involved in the processing of evolutionarily urgent or relevant stimuli.

Few studies have investigated social laterality during spontaneous species-specific encounters in nonhuman primates. Casperd and Dunbar (1996) observed the visual orientation toward conspecifics during aggressive encounters in gelada baboons, reporting left visual preference/right hemisphere dominance. Baraud, Buytet, Bec, and Blois-Heulin (2009) identified a similar pattern in different groups of zoo mangabeys during spontaneous approaching behaviors. High-ranking individuals were approached significantly more frequently from their left than their right visual hemifield, suggesting a facilitation for the rapid identification of facial expressions (see Fernández-Carriba et al., 2002; Hauser, 1993) and a better predictability of behaviors. Rogers and Kaplan (1996) captured lateralized information during the daily social activities in orang-utans, capitalizing on the naturalistic social behaviors of the focal animal. Recent evidence has highlighted the importance of the social environment in modulating behavior and social interactions (e.g., Chartrand \& Bargh, 1999; Forrester, Leavens, Quaresmini, \& Vallortigara, 2011; Forrester, Quaresmini, Leavens, Mareschal, \& Thomas, 2013; Forrester, Quaresmini, Leavens, Spiezio, \& Vallortigara, 2012). Furthermore, Leavens and colleagues (2004) found a right hemisphere dominance manifested by chimpanzees as self-scratching behaviors when task difficulty increased. Although much evidence points to a right hemisphere dominance for processing arousing/social stimuli, the discrepancy in methods across species inhibits direct comparisons between studies.

In the present study we aim to extend the investigation of social laterality within the natural context of nonhuman primates to shed more light on the cerebral lateralization for the processing of social stimuli. Given their phylogenetic closeness to humans and their large repertoire of social activities, great apes represent a valuable animal model to investigate the functional and evolutionary characteristics of the cerebral lateralization for the processing of social stimuli. We observed the spontaneous social behaviors in a biological family group of peer-reared western lowland gorillas ( $G O$ rilla gorilla gorilla) and in a colony of captive zoo-living chimpanzees (Pan troglodytes), particularly focusing on the side of the body exposed to conspecifics that were in close proximity. Unlike previous studies of nonhuman primates (Baraud et al., 2009; 
Casperd \& Dunbar, 1996), we did not consider the right/left visual field, as this kind of measure might be more suitable for animals with laterally placed eyes and small binocular overlap (Robins et al., 1998). Instead, our analysis was focused on the lateral hemifield of the body that the focal subject presented to conspecifics. This study presents a new methodological approach to the investigation of social laterality in primates. We suggest that lateral positioning is likely to reflect a hemispheric specialization for the processing of social stimuli and represents a heightened state of arousal, which would be inherently associated with a preferential visual processing for the detection of faces and facial expressions.

\section{Method}

\section{Subjects and Housing}

We studied a colony of 12 western lowland gorillas (Gorilla gorilla gorilla), made up of a silverback male, seven adult females, and four juveniles (see Table 1), ranging in age from 2-36 years. The group was a biological family, such that the alpha male fathered all juveniles and all individuals have been reared under the care of their conspecifics. These characteristics were considered to be as close as one could come to the social dynamics of a wild group within captivity. The colony was hosted by Port Lympne Wild Animal Park (Kent, United Kingdom) within the "Palace of the Apes," the world's largest gorilla enclosure. The gorillas were considered 'semi free ranging,' in that they moved freely about the large enclosure, which comprised four composite parts: garden, caged upper, caged lower, and inside.

Additionally, we considered a group of nine captive zoo-living chimpanzees (Pan troglodytes), made up of two adult males, five adult females, and two juvenile females (see Table 2), comprising both parent-reared and hand-reared individuals. The enclosure of the chimpanzees was located in the Safari area of Parco Natura Viva (Bussolengo, Italy), which was accessible to visitors only by car. The enclosure consisted of an outdoor environment, an island furnished with two vertical wood towers, small branches, caves, and a small river, which was surrounded by a water dyke $(9 \mathrm{~m}$ wide), to prevent the chimpanzees from escaping. There was also an indoor area for nighttime, which was connected to the outdoor side with two sash doors.
Both gorillas and chimpanzees were provided every day with different food and enrichments, to elicit the manifestation of specie-specific behaviors and cognitively challenge individuals for the improvement of the animal welfare (Carlstead \& Shepherdson, 2000). Water was provided ad libitum for both species. Experimental permits or ethical approvals were not required for either colony, given the noninvasive nature of this study.

\section{Procedure}

We employed a tripod-mounted camera that could be moved to focus on the focal subject, while maintaining a visual angle that also included the proximal social environment. Videos of chimpanzees were collected using a digital video camera (Sony DCRTRV900E) at 24 frames/s, while they were within the external enclosure. Videos of gorillas were collected both outdoors and indoors. For gorillas, we employed two digital video cameras (Panasonic NVGS11B), one with a close view on the focal subject and one with a wider view of the surrounding social environment. Video footage was synchronized using a flash bulb (see Forrester, 2008; Forrester et al., 2011, 2012, 2013). Video streams were later compressed into a single file of 15 frames/s and composed by a top/bottom format for subsequent coding (i.e., focal view in the top and wide view in the bottom). All data collection sessions were unobtrusive and did not interfere with daily spontaneous activities of the individuals.

We used a continuous focal animal sampling method, whereby 10-min sessions of observations were collected for each subject. Each chimpanzee was observed for a total of 90 minutes, comprising 9 sessions. Although an attempt was made to collect an equivalent number of observational sessions for each gorilla, footage time varied from 30 to 100 minutes across subjects, as the nature of the large enclosure, which was enriched with equipment to replicate the natural environment, had the potential to obscure visibility of the focal animal (see Table 1). All video footage was considered to hold valuable contributions to our observational study. As a result of unequal observation times, measures were taken to equalize the weighting of each individual toward the subsequent data set.

Information about lateralized positioning toward conspecifics was collected using a quantitative Multi Dimensional Method

Table 1

Frequencies and z Scores of Sagittal (Front/Rear) and Lateral (Left/Right) Condition in Gorillas

\begin{tabular}{|c|c|c|c|c|c|c|c|c|c|c|}
\hline Subject & Gender & Status & Front & Rear & $z$ score & Left & Right & $z$ score & Total & Minutes $^{\mathrm{a}}$ \\
\hline Dishi & M & $\mathrm{J}$ & 159 & 72 & $-5.66^{b}$ & 137 & 52 & $-6.11^{b}$ & 420 & 90 \\
\hline Djala & $\mathrm{M}$ & $\mathrm{A}$ & 125 & 36 & $-6.93^{b}$ & 28 & 49 & $2.28^{\mathrm{b}}$ & 238 & 90 \\
\hline Emmie & $\mathrm{F}$ & A & 105 & 5 & $-9.44^{b}$ & 45 & 23 & $-2.55^{\mathrm{b}}$ & 178 & 90 \\
\hline Foufou & $\mathrm{F}$ & A & 212 & 261 & $2.20^{\mathrm{b}}$ & 110 & 70 & $-2.90^{\mathrm{b}}$ & 653 & 90 \\
\hline Jaja & $\mathrm{M}$ & $\mathrm{J}$ & 115 & 15 & $-8.68^{b}$ & 54 & 22 & $-3.55^{\mathrm{b}}$ & 206 & 90 \\
\hline Kibi & $\mathrm{F}$ & $\mathrm{A}$ & 28 & 24 & -0.42 & 30 & 15 & $-2.08^{b}$ & 97 & 30 \\
\hline Kishi & $\mathrm{F}$ & A & 84 & 3 & $-8.58^{b}$ & 62 & 32 & $-2.29^{b}$ & 181 & 90 \\
\hline Kouni & $\mathrm{M}$ & $\mathrm{J}$ & 96 & 64 & $-2.45^{b}$ & 76 & 70 & -0.41 & 306 & 90 \\
\hline Mumba & $\mathrm{F}$ & A & 27 & 4 & $-3.95^{\mathrm{b}}$ & 19 & 16 & -0.34 & 66 & 50 \\
\hline Tamarilla & $\mathrm{F}$ & A & 31 & 9 & $-3.32^{b}$ & 34 & 45 & 1.12 & 119 & 100 \\
\hline Tamki & $\mathrm{F}$ & A & 91 & 27 & $-5.80^{b}$ & 46 & 25 & $-2.37^{b}$ & 189 & 90 \\
\hline Yene & $\mathrm{F}$ & $\mathrm{J}$ & 70 & 50 & -1.73 & 101 & 62 & $-2.98^{b}$ & 283 & 80 \\
\hline
\end{tabular}

Note. $\mathrm{M}=$ male, $\mathrm{F}=$ female; $\mathrm{J}=$ juvenile, $\mathrm{A}=$ adult.

${ }^{\mathrm{a}}$ Focal Sampling time in minutes. ${ }^{\mathrm{b}}$ Significant sagittal and lateral asymmetry. 
Table 2

Frequencies and $z$ Scores of Sagittal (Front/Rear) and Lateral (Left/Right) Condition in Chimpanzees

\begin{tabular}{|c|c|c|c|c|c|c|c|c|c|c|}
\hline Subject & Gender & Status & Front & Rear & $z$ score & Left & Right & $z$ score & Total & Minutes $^{\mathrm{a}}$ \\
\hline Camilla & $\mathrm{F}$ & A & 117 & 9 & $-9.53^{b}$ & 3 & 16 & $2.75^{b}$ & 145 & 90 \\
\hline Davidino & M & A & 42 & 12 & $-3.95^{b}$ & 27 & 7 & $-3.26^{b}$ & 88 & 90 \\
\hline Giorgina & $\mathrm{F}$ & $\mathrm{J}$ & 56 & 3 & $-6.77^{b}$ & 56 & 23 & $-3.60^{b}$ & 138 & 90 \\
\hline Giudy & $\mathrm{F}$ & $\mathrm{A}$ & 25 & 5 & $-3.47^{b}$ & 5 & 7 & 0.29 & 42 & 90 \\
\hline Jacky & M & $\mathrm{A}$ & 80 & 3 & $-8.34^{b}$ & 36 & 14 & $-2.97^{b}$ & 133 & 90 \\
\hline Luisa & $\mathrm{F}$ & A & 22 & 0 & $-4.48^{b}$ & 22 & 1 & $-4.17^{b}$ & 45 & 90 \\
\hline Mary & $\mathrm{F}$ & A & 98 & 14 & $-7.84^{\mathrm{b}}$ & 33 & 19 & -1.80 & 164 & 90 \\
\hline Samy & $\mathrm{F}$ & A & 28 & 5 & $-3.83^{b}$ & 58 & 10 & $-5.70^{b}$ & 101 & 90 \\
\hline Valentina & $\mathrm{F}$ & $\mathrm{J}$ & 130 & 12 & $-9.82^{b}$ & 21 & 30 & 1.12 & 193 & 90 \\
\hline
\end{tabular}

Note. $\mathrm{M}=$ male; $\mathrm{F}=$ female; $\mathrm{J}=$ juvenile; $\mathrm{A}=$ adult.

${ }^{a}$ Focal Sampling time in minutes. ${ }^{\mathrm{b}}$ Significant sagittal and lateral asymmetry.

(MDM), first proposed by Forrester (2008) and then employed for studies across species including gorillas (Forrester et al., 2011), chimpanzees (Forrester et al., 2012), and typical preschool children (Forrester et al., 2013). This method is noninvasive and employs a corpus-coding quantitative approach that allows viewing off-line videos streams to extract synchronous and sequential patterns from a distributed database of behavioral activities within a natural context. It is suitable for a reliable quantitative comparison of species-specific and spontaneous behaviors between different primate and nonprimate species. Most importantly, it allows for patterns to be revealed by analyzing fine-grained behaviors from a bottom up perspective.

\section{Data Coding}

The information regarding the positioning of the focal subject toward one or more conspecifics was coded and stored using OBSERVATRON software, which was designed to run on a Mac OS X platform. We distinguished between two main conditions: 1) sagittal condition, which comprised the front and the rear behavioral categories; 2) lateral condition, which comprised the left and the right behavioral categories. More specifically, the sagittal condition referred to when the recipient was positioned in line with the sagittal plane of the focal subject, either in front or behind (in the latter case the recipient was not visible by the focal subject). Alternatively, the lateral condition corresponded to when the recipient was in line with the axial plane of the focal subject, either on its right or left side. Each behavioral category was treated as an independent event, thus when the conspecific resulted in an ambiguous position (i.e., frontal/left, frontal/right, rear/left, rear/ right), the event was discarded from the analysis. Although the head can move independently from the body, head movements were not collected for this investigation. To ensure that the relative presence of the social partner was influencing the positioning of the focal subject, we adopted a conventional distance of $3 \mathrm{~m}$ or less between the focal subject and the conspecifics.

We considered manual activities as a conventional referential event to assess social laterality, because they may require attention by the focal individual. Therefore, a strategic social positioning is likely adopted to facilitate any behavioral responses toward the social environment. Specifically, every time the focal subject performed any manual action with either hand (e.g., collecting an object, self-touching), we coded the physical positions of conspe- cifics that were in close proximity (within $3 \mathrm{~m}$ ) as front, rear, left, or right. If the focal subject was performing a manual activity and a conspecific presented themselves within the $3 \mathrm{~m}$ of the focal individual, the event was not coded as it would not provide information about the active choice of the focal subject. The MDM method allowed simultaneous coding of the focal individual and two conspecifics. Therefore, when there were more than two conspecifics within $3 \mathrm{~m}$ of distance from the focal subject, only the two closest individuals were considered.

\section{Statistical Analysis}

Because analyses were all based on frequencies, to normalize the weighting that each subject contributed to the data set, proportions for each subject of each response type were calculated in relation to the total number of events related to the side positioning for that subject. Moreover, to retain all recorded video of gorilla focal follows, the frequencies were converted into rates (frequency/ minutes of observation) to normalize the weighting of each individual's contribution to the database. To analyze the data set, a two-tailed paired-sample $t$ test was used to assess differences within the sagittal and the lateral conditions, with the level of significance set at .05. To analyze individual-preference for both the sagittal and the lateral condition, we computed a binomial $z$ score for each individual based on the distribution of front/rear and left/right side positioning events. Individuals with scores $>+1.96$ were estimated with a either a rear or right side preference, individuals with scores $<-1.96$ were considered with a either front or left side preference, and individuals with scores in between were considered ambi-preferent (see Table 1).

\section{Results}

\section{Gorillas}

The sagittal condition revealed a greater number of events for the front side (proportions: $M=.403,95 \% \mathrm{CI}=.327-.478$; rates: $M=1.135,95 \% \mathrm{CI}=.793-1.477)$ compared with rear side (proportions: $M=.146,95 \% \mathrm{CI}=.077-.214$; rates: $M=$ $.580,95 \% \mathrm{CI}=.079-1.081)$ and the paired $t$ test confirmed a significant preference for keeping a conspecific to the front (proportions: $t(11)=4.497, p=.001$; rates: $t(11)=3.759, p=$ .003 ; see Figure 1). 


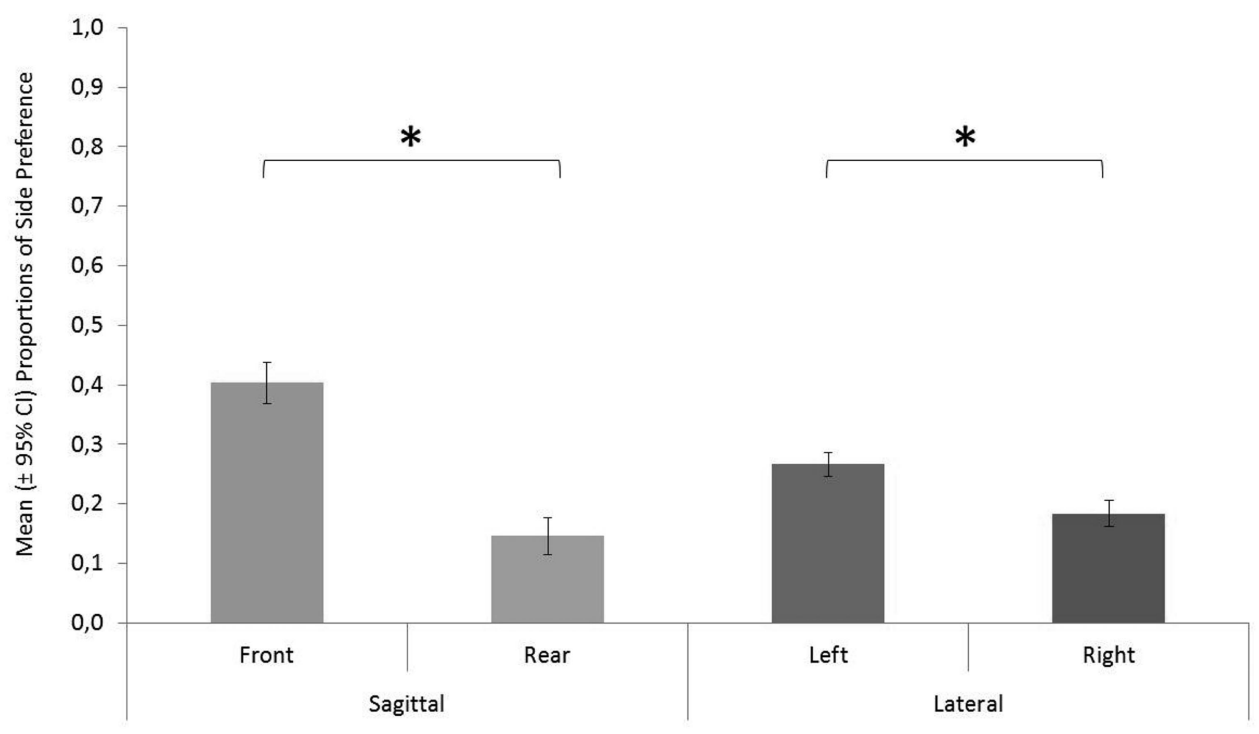

Figure 1. Mean proportion ( $\pm 95 \%$ confidence interval) of gorillas' positioning side preference toward conspecifics. Significant results are highlighted by stars $\left(^{*}\right)$.

The lateral condition also revealed a side bias between the left side (proportions: $M=.267,95 \% \mathrm{CI}=.223-.310$; rates: $M=.765,95 \% \mathrm{CI}=.508-1.022)$ and the right side positioning preference (proportions: $M=.184,95 \% \mathrm{CI}=.135-.232$; rates: $M=.488,95 \% \mathrm{CI}=.357-.619)$, with paired $t$ test revealing a significant preference for keeping a conspecific on left side (proportions: $t(11)=2.973, p=.013$; rates: $t(11)=2.103, p=$ .047 ; see Figure 1).

At the individual level, the binomial $z$ score revealed that 8 of 12 subjects manifested a significant bias for the left and one (the alpha male) for the right side (see Table 1).

\section{Chimpanzees}

The sagittal condition revealed a greater number of events for the front side (proportions: $M=.535,95 \% \mathrm{CI}=.423-.646$; rates: $M=.738,95 \% \mathrm{CI}=.385-1.091)$ compared with rear side (proportions: $M=.061,95 \% \mathrm{CI}=.026-.095$; rates: $M=.078$, $95 \% \mathrm{CI}=.036-.120)$ and the $t$ test confirmed a significant preference for keeping a conspecific frontally (proportions: $t(8)=$ 9.843, $p<.001$; rates $t(8)=4.615, p=.001$; see Figure 2).

The lateral condition was also analyzed by comparing the preference for keeping conspecifics either on the left side (proportions:

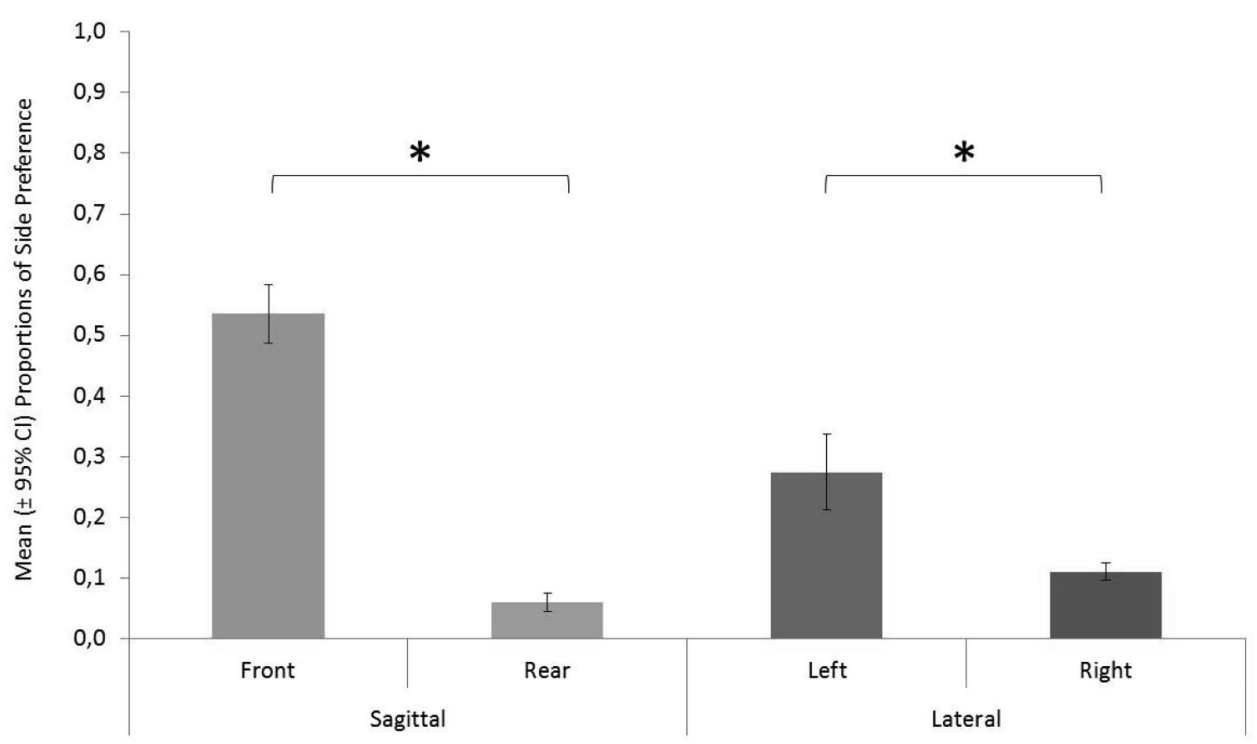

Figure 2. Mean proportion ( $\pm 95 \%$ confidence interval) of chimpanzees' positioning side preference toward conspecifics. Significant results are highlighted by stars $\left(^{*}\right)$. 
$M=.275,95 \% \mathrm{CI}=.132-.417$; rates: $M=.322 ; 95 \% \mathrm{CI}=$ $.156-.488$ ) or the right (proportions: $M=.110,95 \% \mathrm{CI}=.076-$ .143 ; rates: $M=.157,95 \% \mathrm{CI}=.080-.234$ ), which also demonstrated a bias for the left side, although the result just missed significance (proportions: $t(8)=2.339, p=.048$; rates: $t(8)=$ 2.225, $p=.056$; see Figure 2).

At the individual level, the binomial $z$ score revealed that 5 of 8 subjects manifested a significant bias for the left and one for the right side (see Table 2).

\section{Discussion}

The current study investigated, for the first time, the manifestation of sagittal positioning (front/back) and lateralized positioning (left/right) associated with social stimuli in great apes. We analyzed naturalistic encounters using both proportions and rates as a conservative measure to statistically standardize the data set assessment of side preference. A broad-spectrum analysis revealed a population-level preference in keeping conspecifics more to the front rather than behind, in both gorillas and chimpanzees. This outcome is not surprising because keeping social partners positioned in front of the viewer is likely to facilitate the predictability of behaviors and the identification of individuals and their facial expressions. Indeed, nonhuman primates, as humans, are characterized by frontally located eyes, which allow for a stereoscopic vision (Conroy, 1990).

When we considered only the lateral condition (i.e., left vs. right), a significant population-level preference emerged in gorillas to keep conspecifics on the left compared with the right side. Chimpanzees expressed the same pattern of behavioral preference as gorillas at the group level, however statistical tests revealed a trend rather than a statistically significant outcome. We propose that that a left-side bias could reflect an underlying right hemisphere specialization for different cognitive functions that play a critical role in the adaptation of individuals to the social environment.

First, we suggest that exposing the left side more frequently than the right side toward a conspecific could be associated with a right hemisphere specialization to control rapid motor responses to unexpected and dangerous events (MacNeilage et al., 2009; Rogers, 2000a). In this context, exposing the left side of the body to conspecifics might be advantageous during novel or urgent situations to execute physical behaviors for protection and locomotion escape behaviors. These responses may be driven by a left eye dominance to control aggressive behaviors. Several vertebrate species, such as birds (Franklin III \& Lima, 2001; Koboroff, Kaplan, \& Rogers, 2008; Rogers, 2000b), lizards (in the laboratory: Bonati, Csermely, \& Sovrano, 2013; in the wild: Martín, López, Bonati, \& Csermely, 2010), and toads (Lippolis, Bisazza, Rogers, \& Vallortigara, 2002), manifest a left eye preference in monitoring a predator and a rightward preference for escaping from the dangerous stimulus. Additionally, the exposure of the left side of the body toward conspecifics might better facilitate using the hand that is more involved in social interactions, as previously found in gorillas and chimpanzees. In these studies, animate targets elicited equal use of the left and right hands, indicating a higher proportion of left hand actions dedicated to animate compared with inanimate targets (see Forrester et al., 2011, 2012).
Second, the preference to keep conspecifics on the left side is likely to favor the positioning of the left side of the face toward other group members, as previously suggested by Baraud and colleagues (2009), to clearly show the arousal status and prevent extreme aggressive physical reactions. Indeed, the left side of the face of nonhuman primates displays the emotive expression earlier and more intensively than the right side (Fernández-Carriba et al., 2002; Hauser, 1993; Hook-Costigan \& Rogers, 1998).

Third, the preference to keep social partners to the left side might reflect a left-visual advantage for the processing of social stimuli. The use of the left eye to monitor conspecifics is widespread in vertebrates, implying a common evolution of the right hemisphere for the processing of social stimuli and arousing situations (for a review, see Rosa Salva et al., 2012). In humans and great apes, a left bias exists in maternal cradling, thought to be driven, apart from other selective social advantages, by a left visual/right hemisphere preference in the mother to monitor the baby (Hopkins, 2004; Salk, 1960).

Fourth, the preference for keeping conspecifics in the left side could be associated with a greater involvement of the left visual field/right hemisphere in recognizing faces and facial expressions (Morris \& Hopkins, 1993). This outcome is in line with studies considering humans (De Renzi et al., 1994; Kanwisher et al., 1998, although a recent study also reported small contributions from the left hemisphere: Meng, Cherian, Singal, \& Sinha, 2012), monkeys (Broad, Mimmack, \& Kendrick, 2000; Guo et al., 2009; Hamilton \& Vermeire, 1988; Pinsk, DeSimone, Moore, Gross, \& Kastner, 2005), dogs (Guo et al., 2009), and sheep (Peirce et al., 2000; Peirce \& Kendrik, 2002). Furthermore, studies investigating lateralized visual preference to monitor familiar versus unfamiliar conspecifics reported a left eye/right hemisphere preference in the domestic chick (Deng \& Rogers, 2002; Vallortigara \& Andrew, 1991; Vallortigara, Cozzuti, Tommasi, \& Rogers, 2001) and fish (Brown, Western, \& Braithwaite, 2007; Sovrano, 2004). Therefore, gorillas and chimpanzees might prefer to position toward conspecifics preferentially employing their left visual field, to facilitate conspecific identification and monitor other's arousal and behavioral intentions.

The individual-level data analysis revealed that, unlike most gorillas and chimpanzees in the study, the gorilla alpha male and one chimpanzee female manifested a significant bias to keep conspecifics on the right side. A similar finding has been reported during the analysis of visual laterality in the domestic horse while interacting with humans, where, compared with the majority of individuals, some subjects manifested a significant opposite right eye dominance (Farmer, Krueger, \& Byrne, 2010). Further studies are required to elucidate whether social ranking and gender influence lateral positioning. However, a minority of reversed laterality is also known to be present in humans for handedness, that is, $10 \%$ of the entire population is left-handers (Annett, 2002; Steele \& Uomini, 2005). It is documented that in most populations with a directional asymmetry, there is a minority group with an opposite behavioral lateralization, which is less predictable and thus more favored during social aggressive encounters (see Ghirlanda \& Vallortigara, 2004; Vallortigara \& Rogers, 2005).

Overall, the findings of this study suggest that lateralized positioning toward social stimuli might help animals to attend to two different tasks simultaneously, such as foraging or tool use activities, which seems to be controlled by the left hemisphere (for right 
handedness toward inanimate targets in primates, see Forrester et al., 2011, 2012), and vigilance (Rogers et al., 2004). This type of asymmetric behavioral activity might have an adaptive value, facilitating simple reflexive and automatic responses to increase the survival of individuals (Rutherford \& Lindell, 2011). Indeed, approaching and withdrawing processes are at the basis of motor behavior as they are common along the whole animal phylogeny (Davidson, Ekman, Saron, Senulis, \& Friesen, 1990; Quaranta, Siniscalchi, \& Vallortigara, 2007). With the appearance of more complex social systems and interactions in social animals, these behavioral differentiations might have been interpreted as a differentiation in the perception of emotion expression.

We propose that our results may represent a corroboration of the hypothesis that population-level lateralization developed as a byproduct of the social life (Ghirlanda \& Vallortigara, 2004; Vallortigara \& Rogers, 2005), which induced the alignment of individual-level asymmetries to facilitate the social communication and the prediction of social responses. This theoretical proposal is supported by evidence from studies on the domestic chick, demonstrating that typically lateralized individuals (i.e., light incubated) show enhanced performance during a transitive inference task compared to nonlateralized ones (i.e., dark incubated), especially when using their left eye/right hemisphere (Daisley, Regolin, \& Vallortigara, 2010).

The observation of lateralized positioning toward conspecifics might represent a valid behavioral marker for the manifestation of the hemispheric involvement in social interactions. This approach allows for the capture of information associated with the underlying cognitive processing of the social environment without interfering with naturalistic dynamics of individuals. Moreover, the naturalistic conditions of these experiments are useful to facilitate our understanding of how the social environment may modulate physical positioning in primates. We stress the necessity of replicating similar observations in different populations of primates, while collecting more information about other variables that might influence the laterality degree of the social positioning (e.g., rank, gender, reference task, manual activity). Furthermore, we suggest that a similar assessment could be applied to human populations, as the methodology is designed to accommodate laboratory, captive and wild settings alike. Most importantly, it could help to assess emotion processing in human subjects, which have been poorly investigated using an observational approach within a natural setting.

\section{References}

Anfora, G., Rigosi, E., Frasnelli, E., Ruga, V., Trona, F., \& Vallortigara, G. (2011). Lateralization in the invertebrate brain: Left-right asymmetry of olfaction in bumble bee, Bombus terrestris. PLoS One, 6, e18903. doi:10.1371/journal.pone. 0018903

Annett, M. (2002). Handedness and brain asymmetry: The right shift theory. Hove, UK: Psychology Press.

Baraud, I., Buytet, B., Bec, P., \& Blois-Heulin, C. (2009). Social laterality and 'transversality' in two species of mangabeys: Influence of rank and implication for hemispheric specialization. Behavioural Brain Research, 198, 449-458. doi:10.1016/j.bbr.2008.11.032

Bonati, B., Csermely, D., \& Sovrano, V. A. (2013). Looking at a predator with the left or right eye: Asymmetry of response in lizards. Laterality: Asymmetries of Body, Brain and Cognition, 18, 329-339. doi:10.1080/ 1357650X.2012.673623
Borod, J. C., Cicero, B. A., Obler, L. K., Welkowitz, J., Erhan, H. M., Santschi, C., . . . Whalen, J. R. (1998). Right hemisphere emotional perception: Evidence across multiple channels. Neuropsychology, 12, 446-458. doi:10.1037/0894-4105.12.3.446

Braccini, S. N., Lambeth, S. P., Schapiro, S. J., \& Fitch, W. T. (2012). Eye preferences in captive chimpanzees. Animal Cognition, 15, 971-978. doi:10.1007/s10071-012-0523-9

Broad, K. D., Mimmack, M. L., \& Kendrick, K. M. (2000). Is right hemispheric specialization for face discrimination specific to humans? European Journal of Neuroscience, 12, 731-741. doi:10.1046/j.14609568.2000.00934.x

Brown, C., Western, J., \& Braithwaite, V. A. (2007). The influence of early experience on, and inheritance of, cerebral lateralization. Animal Behaviour, 74, 231-238. doi:10.1016/j.anbehav.2006.08.014

Burt, D. M., \& Perret, D. I. (1997). Perceptual asymmetries in judgments of facial attractiveness, age, gender, speech and expression. Neuropsychologia, 35, 685-693. doi:10.1016/S0028-3932(96)00111-X

Campbell, R. (1982). Asymmetries in moving faces. British Journal of Psychology, 73, 95-103. doi:10.1111/j.2044-8295.1982.tb01794.x

Carlstead, K., \& Shepherdson, D. (2000). Alleviating stress in zoo animals with environmental enrichment. In G. P. Moberg \& J. A. Mench (Eds.), The biology of animal stress (pp. 337-354). New York, NY: CABI publishing. doi:10.1079/9780851993591.0337

Casperd, J. M., \& Dunbar, R. I. M. (1996). Asymmetries in the visual processing of emotional cues during agonistic interactions by gelada baboons. Behavioural Processes, 37, 57-65. doi:10.1016/03766357(95)00075-5

Chartrand, T. L., \& Bargh, J. A. (1999). The chameleon effect: The perception-behavior link and social interaction. Journal of Personality and Social Psychology, 76, 893-910. doi:10.1037/0022-3514.76.6.893

Conroy, G. C. (1990). Primate evolution. New York, NY: Norton and Co.

Daisley, J. N., Regolin, L., \& Vallortigara, G. (2010). Logic in an asymmetrical (social) brain: Transitive inference in the young domestic chick. Social Neuroscience, 5, 309-319. doi:10.1080/17470910903529795

Davidson, R. J. (1995). Cerebral asymmetry, emotion, and affective style. In R. J. Davidson \& K. Hugdahl (Eds.), Brain asymmetry (pp. 361-387). Cambridge, MA: MIT Press.

Davidson, R. J., Ekman, P., Saron, C. D., Senulis, J. A., \& Friesen, W. V. (1990). Approach-withdrawal and cerebral asymmetry: Emotional expression and brain physiology I. Journal of Personality and Social Psychology, 58, 330-341. doi:10.1037/0022-3514.58.2.330

Deckel, A. W. (1995). Lateralization of aggressive responses in Anolis. Journal of Experimental Zoology, 272, 194-200. doi:10.1002/jez .1402720304

de Latude, M., Demange, M., Bec, P., \& Blois-Heulin, C. (2009). Visual laterality responses to different emotive stimuli by red-capped mangabeys, Cercocebus torquatus torquatus. Animal Cognition, 12, 31-42. doi:10.1007/s10071-008-0166-z

Deng, C., \& Rogers, L. J. (2002). Social recognition and approach in the chick: Lateralization and effect of visual experience. Animal Behaviour, 63, 697-706. doi:10.1006/anbe.2001.1942

De Renzi, E., Perani, D., Carlesimo, G. A., Silveri, M. C., \& Fazio, F. (1994). Prosopagnosia can be associated with damage confined to the right hemisphere: An MRI and PET study and a review of the literature. Neuropsychologia, 32, 893-902. doi:10.1016/0028-3932(94)90041-8

Farmer, K., Krueger, K., \& Byrne, W. B. (2010). Visual lateralization in the domestic horse (Equus caballus) interacting with humans. Animal Cognition, 13, 229-238. doi:10.1007/s10071-009-0260-x

Fernández-Carriba, S., Loeches, A., Morcillo, A., \& Hopkins, W. D. (2002). Functional asymmetry of emotions in primates: New findings in chimpanzees. Brain Research Bulletin, 57, 561-564. doi:10.1016/ S0361-9230(01)00685-2 
Forrester, G. S. (2008). A multidimensional approach to investigations of behaviour: Revealing structure in animal communication signals. Animal Behaviour, 76, 1749-1760. doi:10.1016/j.anbehav.2008.05.026

Forrester, G. S., Leavens, D. A., Quaresmini, C., \& Vallortigara, G. (2011). Target animacy influences gorilla handedness. Animal Cognition, 14, 903-907. doi:10.1007/s10071-011-0413-6

Forrester, G. S., Quaresmini, C., Leavens, D. A., Mareschal, D., \& Thomas, M. S. C. (2013). Human handedness: An inherited evolutionary trait. Behavioural Brain Research, 237, 200-206. doi:10.1016/j.bbr 2012.09.037

Forrester, G. S., Quaresmini, C., Leavens, D. A., Spiezio, C., \& Vallortigara, G. (2012). Target animacy influences chimpanzee handedness. Animal Cognition, 15, 1121-1127. doi:10.1007/s10071-012-0536-4

Franklin III, W. E., \& Lima, S. L. (2001). Laterality in avian vigilance: Do sparrows have a favourite eye? Animal Behaviour, 62, 879-885. doi: 10.1006/anbe.2001.1826

Frasnelli, E., Vallortigara, G., \& Rogers, L. J. (2012). Left-right asymmetries of behaviour and nervous system in invertebrates. Neuroscience \& Biobehavioral Reviews, 36, 1273-1291. doi:10.1016/j.neubiorev.2012 .02 .006

Ghirlanda, S., Frasnelli, E., \& Vallortigara, G. (2009). Intraspecific competition and coordination in the evolution of lateralization. Philosophical Transactions of the Royal Society B: Biological Sciences, 364, 861-866. doi:10.1098/rstb.2008.0227

Ghirlanda, S., \& Vallortigara, G. (2004). The evolution of brain lateralization: A game theoretical analysis of population structure. Proceedings of the Royal Society B: Biological Sciences, 271, 853-857. doi:10.1098/ rspb.2003.2669

Guo, K., Meints, K., Hall, C., Hall, S., \& Mills, D. (2009). Left gaze bias in humans, rhesus monkeys and domestic dogs. Animal Cognition, 12, 409-418. doi:10.1007/s10071-008-0199-3

Hamilton, C. R., \& Vermeire, B. A. (1988). Complementary hemispheric specialization in monkeys. Science, 242, 1691-1694. doi:10.1126/ science. 3201258

Hauser, M. D. (1993). Right hemisphere dominance for the production of facial expression in monkeys. Science, 261, 475-477. doi:10.1126/ science. 8332914

Hews, D. K., \& Worthington, R. A. (2001). Fighting from the right side of the brain: Left visual field preference during aggression in free-ranging male lizards (Urosaurus ornatus). Brain, Behavior and Evolution, 58, 356-361. doi:10.1159/000057576

Hook-Costigan, M. A., \& Rogers, L. J. (1998). Lateralized use of the mouth in production of vocalizations by marmosets. Neuropsychologia, 36, 1265-1273. doi:10.1016/S0028-3932(98)00037-2

Hopkins, W. D. (2004). Laterality in maternal cradling and infant positional biases: Implications for the development and evolution of hand preferences in nonhuman primates. International Journal of Primatology, 25, 1243-1265. doi:10.1023/B:IJOP.0000043961.89133.3d

Kanwisher, N., Tong, F., \& Nakayama, K. (1998). The effect of face inversion on the human fusiform face area. Cognition, 68, B1-B11. doi:10.1016/S0010-0277(98)00035-3

Karenina, K., Giljov, A., Baranov, V., Osipova, L., Krasnova, V., \& Malashichev, Y. (2010). Visual laterality of calf-mother interactions in wild whales. PLoS One, 5, e13787. doi:10.1371/journal.pone.0013787

Koboroff, A., Kaplan, G., \& Rogers, L. J. (2008). Hemispheric specialization in Australian magpies (Gymnorhina tibicen) shown as eye preferences during response to a predator. Brain Research Bulletin, 76, 304306. doi:10.1016/j.brainresbull.2008.02.015

Leavens, D. A., Aureli, F., \& Hopkins, W. D. (2004). Behavioral evidence for the cutaneous expression of emotion in a chimpanzee (Pan troglodytes). Behaviour, 141, 979-997. doi:10.1163/1568539042360189

Levy, J. (1977). The mammalian brain and the adaptive advantage of cerebral asymmetry. Annals of the New York Academy of Sciences, 299, 264-272. doi:10.1111/j.1749-6632.1977.tb41913.x
Lippolis, G., Bisazza, A., Rogers, L. J., \& Vallortigara, G. (2002). Lateralisation of predator avoidance responses in three species of toads. Laterality: Asymmetries of Body, Brain and Cognition, 7, 163-183. doi:10.1080/13576500143000221

MacNeilage, P. F., Rogers, L. J., \& Vallortigara, G. (2009). Origins of the left and right brain. Scientific American, 301, 60-67. doi:10.1038/ scientificamerican0709-60

Martín, J., López, P., Bonati, B., \& Csermely, D. (2010). Lateralization when monitoring predators in the wild: A left eye control in the common wall lizard (Podarcis muralis). Ethology, 116, 1226-1233. doi:10.1111/ j.1439-0310.2010.01836.x

Meng, M., Cherian, T., Singal, G., \& Sinha, P. (2012). Lateralization of face processing in the human brain. Proceedings of the Royal Society B: Biological Sciences, 279, 2052-2061. doi:10.1098/rspb.2011.1784

Morris, R. D., \& Hopkins, W. D. (1993). Perception of human chimeric faces by chimpanzees: Evidence for a right hemisphere advantage. Brain and Cognition, 21, 111-122. doi:10.1006/brcg.1993.1008

Nagy, M., Àkos, Z., Biro, D., \& Vicsek, T. (2010). Hierarchical group dynamics in pigeon flocks. Nature, 464, 890-893. doi:10.1038/ nature 08891

Peirce, J. W., \& Kendrik, K. M. (2002). Functional asymmetry in sheep temporal cortex. Neuroreport, 13, 2395-2399. doi:10.1097/00001756200212200-00004

Peirce, J. W., Leigh, A. E., \& Kendrick, K. M. (2000). Configurational coding, familiarity and the right hemisphere advantage for face recognition in sheep. Neuropsychologia, 38, 475-483. doi:10.1016/S00283932(99)00088-3

Pinsk, M. A., DeSimone, K., Moore, T., Gross, C. G., \& Kastner, S. (2005). Representations of faces and body parts in macaque temporal cortex: A functional MRI study. PNAS Proceedings of the National Academy of Sciences of the United States of America, 102, 6996-7001. doi:10.1073/ pnas.0502605102

Quaranta, A., Siniscalchi, M., \& Vallortigara, G. (2007). Asymmetric tail-wagging responses by dogs to different emotive stimuli. Current Biology, 17, 199-201. doi:10.1016/j.cub.2007.02.008

Robins, A., Lippolis, G., Bisazza, A., Vallortigara, G., \& Rogers, L. J. (1998). Lateralized agonistic responses and hindlimb use in toads. Animal Behaviour, 56, 875-881. doi:10.1006/anbe.1998.0877

Rogers, L. J. (2000a). Advantages and disadvantages of lateralization. In L. J. Rogers \& R. J. Andrew (Eds.), Comparative vertebrate lateralization (pp. 126-153). Cambridge, UK: Cambridge University Press.

Rogers, L. J. (2000b). Evolution of hemispheric specialization: Advantages and disadvantages. Brain and Language, 73, 236-253. doi:10.1006/brln .2000 .2305

Rogers, L. J., \& Andrew, R. J. (2002). Comparative vertebrate lateralization. Cambridge, UK: Cambridge University Press. doi:10.1017/ CBO9780511546372

Rogers, L. J., \& Kaplan, G. (1996). Hand preferences and other lateral biases in rehabilitated orang-utans, Pongo pygmaeus pygmaeus Animal Behaviour, 51, 13-25. doi:10.1006/anbe.1996.0002

Rogers, L. J., Vallortigara, G., \& Andrew, R. J. (2013). Divided brains. The biology and behaviour of brain asymmetries. New York, NY: Cambridge University Press. doi:10.1017/CBO9780511793899

Rogers, L. J., Zucca, P., \& Vallortigara, G. (2004). Advantages of having a lateralized brain. Proceedings of the Royal Society B: Biological Sciences, 271, 420-422. doi:10.1098/rsbl.2004.0200

Rosa Salva, O., Regolin, L., Mascalzoni, E., \& Vallortigara, G. (2012). Cerebral and behavioural asymmetry in animal social recognition. Comparative Cognition \& Behavior Reviews, 7, 110-138. doi:10.3819/ccbr 2012.70006

Rutherford, H. J. V., \& Lindell, A. K. (2011). Author reply: More than evaluation: Lateralization of the neural substrates supporting approach and avoidance motivational systems. Emotion Review, 3, 347-348. doi: $10.1177 / 1754073911402404$ 
Salk, L. (1960). The effects of normal heartbeat sound on the behavior of the newborn infant: Implications for mental health. World Mental Health, 12, 168-175.

Sovrano, V. A. (2004). Visual lateralization in response to familiar and unfamiliar stimuli in fish. Behavioural Brain Research, 152, 385-391. doi:10.1016/j.bbr.2003.10.022

Sovrano, V., Bisazza, A., \& Vallortigara, G. (2001). Lateralization of response to social stimuli in fishes: A comparison between different methods and species. Physiology \& Behavior, 74, 237-244. doi:10.1016/ S0031-9384(01)00552-2

Steele, J., \& Uomini, N. (2005). Humans, tools, and handedness. In V. Roux \& B. Bril (Eds.), Stone knapping: The necessary conditions for a uniquely hominin behaviour (pp. 217-239). Cambridge, UK: McDonald Institute for Archaeological Research.

Vallortigara, G. (1992). Right hemisphere advantage for social recognition in chicks. Neuropsychologia, 30, 761-768. doi:10.1016/00283932(92)90080-6

Vallortigara, G., \& Andrew, R. J. (1991). Lateralization of response to change in a model partner by chicks. Animal Behaviour, 41, 187-194. doi:10.1016/S0003-3472(05)80470-1
Vallortigara, G., Cozzuti, C., Tommasi, L., \& Rogers, L. J. (2001). How birds use their eyes: Opposite left-right specialization for the lateral and frontal visual hemifield in the domestic chick. Current Biology, 11, 29-33. doi:10.1016/S0960-9822(00)00027-0

Vallortigara, G., \& Rogers, L. J. (2005). Survival with an asymmetrical brain: Advantages and disadvantages of cerebral lateralization. Behavioral and Brain Sciences, 28, 575-589. doi:10.1017/ S0140525X05000105

Wallez, C., \& Vauclair, J. (2011). Right hemisphere dominance for emotion processing in baboons. Brain and Cognition, 75, 164-169. doi: 10.1016/j.bandc.2010.11.004

Received July 8, 2013

Revision received February 4, 2014 Accepted February 10, 2014 